
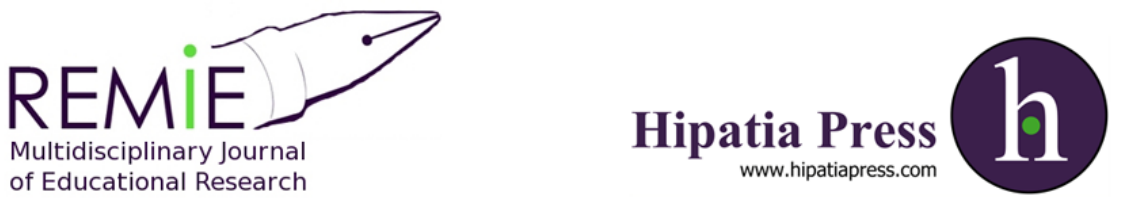

Instructions for authors, subscriptions and further details:

http://remie.hipatiapress.com

\title{
Explorando las Causas del Descenso en la Curiosidad Intelectual durante la Formación Docente en Chile
}

Constanza Lobos1, Carla Muñoz2, Jorge Valenzuela2

1) Universidad Alberto Hurtado

2) Universidad Católica del Maule

Date of publication: February 15th, 2020

Edition period: October 2019-February 2020

To cite this article: Lobos, C., Muñoz, C., \& Valenzuela, J. (2020).

Explorando las Causas del Descenso en la Curiosidad Intelectual durante la Formación Docente en Chile. Multidisciplinary Journal of Educational Research, 10(1), 26-46. doi: 10.4471/remie.2020.4898

To link this article: http://dx.doi.org/10.447/remie.2012.07

\section{PLEASE SCROLL DOWN FOR ARTICLE}

The terms and conditions of use are related to the Open Journal System and to Creative Commons Attribution License (CC-BY). 


\title{
Exploring the Causes of the Drop in Intellectual Curiosity during Teacher Education in Chile
}

Constanza Lobos

Universidad Alberto Hurtado
Carla Muñoz

Universidad Católica del

Maule

Jorge Valenzuela

Universidad Católica del Maule

(Received: 18th November 2019; Accepted: 18th January 2020; Published: 15th February 2020)

\begin{abstract}
Recent research shows significant motivational changes during teacher training including a significant drop in the intellectual curiosity of teachers in training. Given the relevance of this observation and its possible impact on the quality of the training of future teachers, this study aims to describe and analyze student-attributed caused to declined intellectual curiosity during initial teacher training. From a general sample of future primary teacher $(\mathrm{n}=295)$ who were evaluated across motivational variables, students who showed a significant drop in this variable were selected for interview (posttest-pretest delta $\geq-1 \mathrm{z})$. Students were interviewed in depth $(\mathrm{n}=10)$ to explore possible causes behind motivational decline. Causes attributed to low motivation focus on factors within teacher training: expectations/reality discrepancy, lowdemanding or non-challenging environments, and technical conceptions of the teaching profession. Self-reported attributions to reasons behind declining motivation are consistent with previous studies. A generalized perception of low quality, lack of challenge, and the eminently technical character of the profession conspire against student commitment to solid academic formation. Given that intellectual curiosity is key in learning and can provide teachers the opportunity to model the desire to learn in their students, widespread perception of low quality and demand is a wake-up call for training institutions.
\end{abstract}

Keywords: motivation, teacher education, preservice teacher, demotivation, intellectual curiosity 


\section{Explorando las Causas del Descenso en la Curiosidad Intelectual durante la Formación Docente en Chile}

Constanza Lobos

Universidad Alberto Hurtado

Jorge Valenzuela
Carla Muñoz

Universidad Católica del

Maule

\section{Universidad Católica del Maule}

(Recibido: 18 Noviembre 2018; Aceptado: 18 Enero 2020; Publicado: 15 Febrero 2020)

\section{Resumen}

Investigaciones recientes muestran procesos de desmotivación durante la formación profesional, entre ellos una baja en la curiosidad intelectual. Dada la relevancia de esta constatación el presente estudio tiene por objetivo describir y analizar las causas atribuidas por los estudiantes a este fenómeno. A partir de una muestra general de futuros profesores de primaria $(\mathrm{n}=295)$, se seleccionaron estudiantes que evidenciaron una caída significativa en esta variable (delta post-pretest $\geq-1 z$ ). A estos estudiantes les fueron realizadas entrevistas en profundidad $(n=10)$ para explorar las eventuales causas que explicarían esta baja motivacional. Los resultados muestran que las causas atribuidas a esta baja motivacional se focalizan en factores al interior de la formación docente: discordancia expectativa/realidad, ambientes de baja exigencia y poco desafiantes y a una concepción técnica de la profesión docente. La percepción generalizada de baja calidad y exigencia y el carácter eminentemente técnico de la profesión conspiran contra el compromiso de los estudiantes por una formación académica sólida. Dado que la curiosidad intelectual es clave en el aprendizaje y en el profesor puede significar la oportunidad de modelar ese deseo de saber en sus estudiantes, esta percepción generalizada de baja calidad y exigencia es un llamado de alerta a las instituciones formadoras.

Palabras clave: motivación, formación de profesores, estudiantes de pedagogía, desmotivación, curiosidad intelectual 



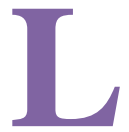

a curiosidad intelectual es un pilar básico del aprendizaje y del desempeño académico (Von Stumm, Hell, \& Chamorro-Premuzic, 2011). El mediador de esta dimensión en contexto escolar es precisamente el profesor. A él le corresponde modelar qué significa aprender y estimular en sus alumnos el placer de aprender. Una baja en esta dimensión, claramente tendrá impacto en el aprendizaje de los estudiantes. Esta indagación se enmarca dentro de un estudio mayor (Valenzuela et al., 2018), en el cual se constató un descenso significativo en la curiosidad intelectual de los estudiantes de la muestra. En este contexto, la presente investigación constituye un estudio exploratorio para identificar cuáles serían las eventuales causas de esta merma.

\section{La Curiosidad Intelectual}

La curiosidad es definida como un viejo, intrigante e incluso incómodo/molesto (vexing) constructo en psicología de la motivación (Silvia, 2012). Múltiples son las definiciones de curiosidad intelectual (cf. Noordewier \& van Dijk, 2017), pero es posible definirle como el deseo de saber (Silvia \& Kashdan, 2009), como un apetito (Berlyne, 1954) o un deseo de conocer el mundo, de sentir placer al realizar esta actividad y proyectar ese deseo en el futuro, que refleja un estado en el que las personas carecen de información y están motivadas para descubrir qué es.

Como variable, la curiosidad intelectual es una medida de la intensidad con que se disfruta una actividad (e.g. aprender cosas nuevas) y corresponde a un tipo de motivación que podemos clasificar dentro de una orientación intrínseca por su alto grado de autodeterminación (Ryan \& Deci, 2017). Esta noción ha sido identificada como una de las disposiciones clave para el pensamiento crítico en donde la búsqueda de estos nuevos conocimientos va más allá de una búsqueda pragmática o utilitarista (Demirdag, 2015; Powell, Nettelbeck, \& Burns, 2016; Watson, 2017). La curiosidad, el interés y la motivación intrínseca son elementos clave en el desarrollo de la competencia, el conocimiento y el expertise (Silvia \& Kashdan, 2009).

Existiría un acuerdo en que la Escuela debería promover en los estudiantes el deseo y el placer por aprender, así como las habilidades para hacerlo (Day \& Gu, 2012; Delors, 2013; Delors et al., 1996). En ello, la curiosidad 
intelectual es un punto clave. Esta "sed de conocimiento" no solo facilita el aprendizaje escolar, sino que permite una comprensión más rica del mundo, o dicho de otra manera, un incremento del capital cognitivo (cf. Rosas \& Santa Cruz, 2013). En la misma línea, la curiosidad intelectual se correlaciona positivamente con estrategias autorregulatorias del aprendizaje (Lauriola et al., 2015) y es una dimensión clave, tanto de alumnos talentosos (Flanagan \& Arancibia, 2005; Silverman, 1997) como también de profesores “excelentes" o "efectivos" (Sanz, Bertolín, \& Lujan, 2017; Siegle, Rubenstein, \& Mitchell, 2014).

Las investigaciones respecto de la relación entre curiosidad intelectual y desempeño académico no son concluyentes. Mientras estudios como los de Costa y Kallick (2009) o Von Stumm et al., (2011) sugieren un efecto positivo de la curiosidad intelectual en los aprendizajes; otros como el de Powell, Nettelbeck y Burns (2017), muestran que el aporte incremental de la curiosidad intelectual, cuando se controla por inteligencia (cristalizada), podría no ser tan significativa para explicar los desempeños académicos.

Con todo, la mayor parte de la literatura revisada aborda la curiosidad intelectual desde una perspectiva prescriptiva, es decir, como un "deber ser" más que como objeto de estudio concreto. Así, encontramos diversos autores que sitúan esta variable como un eje clave, pero sin llegar a operacionalizarla ni a estudiarla en detalle en los maestros, ya sean estos futuros profesores o maestros en ejercicio.

\section{Curiosidad Intelectual y Profesores}

La promoción de la curiosidad intelectual en contexto escolar es atribuida habitualmente, y con razón, al profesor. Se la reconoce como una de las características de los profesores excelentes (Sanz et al., 2017). En primer lugar, porque al maestro le corresponde un rol fundamental de la mediación de la cultura (Falardeau \& Simard, 2007a, 2007b; Zakhartchouk, 1999). Es el maestro quien tiene los conocimientos que permiten contextualizar y asignar sentido a los contenidos escolares y, a través de ellos, mostrar un mundo más complejo que difícilmente el alumno visualiza por sí solo. Aún a pesar de que muchas veces esta responsabilidad sea delegada a la familia (Precht, Valenzuela, Muñoz, \& Sepúlveda, 2016), especialmente en 


\section{Lobos, Muñoz, \& Valenzuela-Curiosidad Intelectual}

profesores que ven su profesión desde una perspectiva más técnica (Oliva, 2017), es el docente quien tiene (o debería tener) el conocimiento para situar los contenidos escolares dentro de una forma más rica de comprender el mundo.

En segundo lugar, la curiosidad intelectual es clave en el profesor ya que el maestro tiene la oportunidad de modelar ese deseo de saber. Tal como plantea Viau (2009; 2013), el profesor es eventualmente el único modelo de "aprendiz" al que tienen acceso los estudiantes (Bouffard, Mariné, \& Chouinard, 2004). Si bien excepcionalmente un estudiante puede acceder a modelos de aprendiz dentro de su entorno, el sistema educativo debiera poder ofrecer las mismas oportunidades de desarrollo a todos sus estudiantes. El problema sobreviene cuando el profesor, quien tiene este desafío, no lo aprovecha ya sea porque concibe su profesión de manera "técnica", fundamentalmente como un transmisor de contenidos (Todorescu, PopescuMitroi, \& Greculescu, 2015) o porque él mismo no ha desarrollado este placer por comprender el mundo de manera más plena. Así, sobre la base de este gusto por saber más, los maestros son capaces de dar testimonio del gusto y pasión por aprender, y a través de ello, suscitar en los alumnos el deseo de aprender (Costa \& Kallick, 2009). De esta manera, un profesor que no ha desarrollado el gusto por aprender y, asimismo, no manifiesta una curiosidad por saber más, podría ser un obstáculo durante su propia formación disciplinar, además de presentar dificultades a la hora de transmitir este placer a sus estudiantes.

\section{Consecuencias de la falta de modelos de aprendices o profesores con baja curiosidad intelectual}

Constatar una baja de la curiosidad intelectual en los futuros profesores no sería buena noticia. Como señalamos anteriormente, si el profesor no imprime en sus alumnos este placer por conocer más, de disfrutar con el aprendizaje, estamos privando a los estudiantes de motivaciones que les permitan activar sus recursos cognitivos para aprender en la Escuela (Costa \& Kallick, 2009).

Lo anterior no es sólo una posibilidad. Investigaciones recientes de nuestro equipo muestran una baja significativa en la Curiosidad Intelectual 
ya en el paso de primer a segundo año de carrera $t(1,113)=2.14 ; p=.03 ; d=-.20$. Esto se traduce en una reducción en la variable Curiosidad Intelectual en un $38 \%$ de los estudiantes (Valenzuela et al., 2018). Este cambio es explicado, en parte, por un efecto de regresión a la media $r_{2}=.245, p<.01$, que explica un $24,5 \%$ de la varianza. No obstante, el restante $75 \%$ no es atribuible a dicho fenómeno y, por lo tanto, es necesario buscar las causas de este porcentaje de variabilidad en la curiosidad intelectual en otros factores.

Una vía posible de exploración se relacionaría con el discurso de los profesores en torno a la importancia de los aspectos prácticos por sobre aquellos más conceptuales o teóricos que informan la profesión, incluso llegando a un cierto desprecio por estos últimos (cf. Cuadra \& Catalán, 2016). Suponemos que esta desvalorización de lo teórico podría ser una pista importante para explicar esta baja en el deseo de saber.

Con todo, un paso inicial para despejar esta incógnita es identificar las posibles causas de esta desmotivación. Para ello, una primera aproximación es caracterizar las causas atribuidas por los estudiantes a esta disminución en la curiosidad intelectual. Evidentemente, esto corresponde a una atribución causal de parte del estudiante que pudiera omitir otros factores de los cuales se es inconsciente. No obstante, estimamos que este es un ejercicio necesario.

\section{Método}

\section{Participantes}

En la fase inicial de este estudio participaron estudiantes de programas de formación pedagógica para futuros profesores de primaria $(n=295)$ de cinco universidades chilenas ubicadas entre las regiones IV y IX. La muestra estuvo compuesta por estudiantes de primer año $(n=114)$ y tercer año $(n=$ 181), es decir, de la etapa inicial e intermedia de la formación pedagógica. Aunque la muestra pudiera parecer sesgada hacia las mujeres $(88,1 \%$ de mujeres), este porcentaje es similar a la proporción nacional de mujeres que estudian este tipo de programa (Servicio de Información de Educación Superior, 2016).

La edad media de los participantes fue de $21.4(s d=3.44) .12 .9 \%$ de los participantes tenía una beca por mérito académico nacional denominada Beca Vocación de Profesor (MINEDUC, 2019), y el 71.2\% había tenido 


\section{Lobos, Muñoz, \& Valenzuela-Curiosidad Intelectual}

experiencia práctica temprana o se había sometido a una actividad introductoria práctica dentro del sistema escolar.

A partir de esta muestra general se seleccionó una sub-muestra para la fase de entrevistas (E), la cual estuvo constituida por un grupo de estudiantes que evidenciaron un descenso significativo y que los situaban a más de una desviación estándar bajo la media, en la variable curiosidad intelectual ( $\geq$ 1z). El número inicial de casos fue de seis estudiantes, incrementándose hasta alcanzar saturación teórica (Ardila \& Rueda, 2013). Finalmente, fueron entrevistados 10 estudiantes con estas características.

\section{Instrumentos}

Para medir Curiosidad Intelectual se utilizó la sub-escala curiosidad intelectual del Inventario Motivacional para la Formación Docente (Valenzuela et al., 2016). Esta escala, inspirada en el trabajo de Facione y equipo (González, 2006), está compuesta de 4 ítems tipo Lickert y refiere a la curiosidad y el deseo de aprender. Ejemplo de ítem es "me encanta aprender cosas nuevas". El instrumento fue sometido a análisis factorial confirmatorio $\left(\chi_{2}=11.58 ; p<.01 ; C F I=.99 ; R M S E A=.07, C I[.03-.13]\right)$ para testear su estructura factorial unidimensional y al cálculo de confiabilidad compuesta $(C R=.72)$

Para la fase de entrevistas se elaboró un guion en donde se les indicaba a los participantes que los resultados generales del estudio habían arrojado una baja significativa en esta variable y se les pedía su parecer sobre las causas que podrían haber ocasionado esta baja en la curiosidad intelectual. Tras su respuesta, se le señalaba que ellos mismos eran parte del grupo que más había cambiado y se les preguntaba si reconocían en ellos este cambio y, en su caso, cómo explicarían esta baja significativa en esta variable.

\section{Procedimientos}

El estudio original (Valenzuela et al., 2018) tuvo por objetivo medir una serie de variables motivacionales con el fin de seguir las trayectorias motivacionales de futuros docentes de primaria. La aplicación en la muestra general fue realizada en dos años consecutivos a través de dos aplicaciones con un desfase de 7 meses (septiembre y mayo), es decir cuando los estudiantes de primero ya cursaban su segundo año y los de tercero, su cuarto 
año.

La selección de casos para las entrevistas se realizó a partir de los cambios pre-post en la variable curiosidad intelectual, invitando a estudiantes que hubiesen tenido una baja igual o mayor a una desviación estándar (delta $\geq$ 1z).

Las entrevistas se realizaron de manera individual, a partir de un guion semiestructurado previamente validado por expertos, y fueron registradas en audio, para luego ser transcritas y analizadas. A los participantes se les recompensó con un par de entradas al cine. En todos los casos, la aprobación ética para la recopilación de datos se obtuvo tanto de las universidades participantes, como de los propios participantes.

\section{Procedimientos Analíticos}

El material transcrito fue codificado por tres codificadores independientes, quienes, a partir de los discursos de los estudiantes, levantaron categorías que permitieran dar cuenta de las razones por las cuales esta variable descendería de manera significativa siete meses después. Esto permitió triangular los análisis y ajustar las categorías de análisis finales y a partir de ellos, realizar un análisis de contenido de las causas aludidas como explicación a este cambio motivacional.

\section{Resultados}

Uno de los hallazgos iniciales y que da contexto a los diferentes motivos por los cuales decrece la curiosidad intelectual durante la formación de futuros profesores es que este proceso ocurre en el contexto de la formación universitaria. Se descarta así, por ejemplo, que la fuente de este descenso motivacional ocurra influido por la Escuela u otros contextos. Las entrevistas revelan que un $94,8 \%$ (55 de 58) de las menciones realizadas respecto a factores de desmotivación de los estudiantes, corresponden a elementos que dicen relación con el contexto de formación profesional universitaria, mientras que solo el 5,2\% restante alude a factores generales o personales sin conexión con esta etapa de formación.

Ahora bien, ¿qué factores de desmotivación propios de la formación universitaria es posible reconocer en el discurso de los estudiantes 


\section{Lobos, Muñoz, \& Valenzuela-Curiosidad Intelectual}

entrevistados?

\section{Tensión Expectativa-Realidad}

Según lo relatado por los entrevistados, existiría una especie de desencanto con la carrera de Pedagogía en diversos ámbitos. Algunos de ellos señalan que la baja en la curiosidad intelectual radicaría en la discordancia entre las expectativas con las que entraron a la universidad y con lo que encontraron durante su estadía en ella. Existiría una suerte de idealización de la Universidad por parte de los estudiantes, en cuanto a contenidos, exigencia, ambiente y aprendizajes; que no necesariamente se condicen con la realidad percibida por ellos durante su formación.

Esta discrepancia entre las expectativas personales y el contexto universitario percibido por el estudiante, se traduce en un juicio crítico frente al nivel de exigencia y desafío inferior al presupuestado, un énfasis en aspectos técnicos por sobre un conocimiento profesional que se concreta en contenidos considerados limitados y en la falta de proposiciones innovadoras a nivel pedagógico.

Era volver a hacer lo mismo una y otra vez. Tampoco te hacían cuestionarte algunas cosas, por querer aprender más o por inventar cosas nuevas, indagar en nuevos estudios para innovar con tus alumnos. ... era como repetir lo mismo de siempre en la práctica (E7).

Además, los estudiantes indican que existiría la sensación de que la Universidad sería, en la práctica, una suerte de continuación de la educación secundaria sin mayores cambios en términos de exigencia respecto a la experiencia escolar. Los estudiantes no notan mayores diferencias entre una etapa y otra, es decir, la universidad no representaría mayores desafíos para los estudiantes, lo que mermaría su motivación por saber más.

La observación según la cual "la universidad ha sido una extensión del colegio" se basa en una mirada crítica a la escolarización de la formación en donde está ausente "este cuestionamiento constante de la educación, de cómo queremos educar y qué queremos buscar en realidad". Esta falta de reflexión más profunda sobre el sentido de la acción profesional, finalmente, "no alimenta este bichito del interés" (E1).

En estos discursos, los estudiantes destacan un interés por problematizar 
la pedagogía y la educación desde la formación docente, interés que no se ve satisfecho en la carrera. La inexistencia de asignaturas que permitan reflexionar sobre el quehacer pedagógico y la educación en general (como filosofía, historia de la educación, sociología, entre otras) -y que sí se impartían en el pasado- podría ir en desmedro de la curiosidad intelectual de los futuros docentes. Dentro de la formación pedagógica, los estudiantes desean espacios de reflexión que no están siendo satisfechos por la carrera.

Creo que la universidad ha ido quitando estos ramos en los cuales se podía ver que el cuestionamiento de la pedagogía era una cosa transversal, o sea, era un aspecto social, era un aspecto cultural, era un aspecto intelectual, creo que esos se han ido quitando dentro de la universidad (E2).

Los estudiantes llegan a la universidad con expectativas acerca de lo que se encontrarán en su programa de formación. No obstante, durante su primer año de formación sus expectativas decaen al encontrarse con un panorama diferente en cuanto a exigencias, aprendizaje de nuevos contenidos, reflexión crítica respecto de la pedagogía y del acontecer nacional en el ámbito educativo. Esta discordancia entre expectativas y realidad de la vida universitaria y la desazón académica, se concreta en el poco o nulo esfuerzo que declaran los entrevistados frente a las tareas académicas requeridas por la formación pedagógica.

Sí, además de que también uno empieza a decir 'este ramo, no sé, puedo responder la prueba solo con lo que aprendí en clases' [...] por ejemplo, para una prueba uno podía llegar ahí sin estudiar nada y podías responderla y no te iba a ir mal en la prueba [...] es un ramo que uno puede enfrentarse con los conocimientos que uno tiene y vas adquiriendo en las clases, pero no creo que sea tan necesario un estudio. No es como tanta dedicación (E10).

\section{Contexto Académico Poco Desafiante}

Un segundo elemento que aparece como causa de la baja en la curiosidad intelectual es la percepción de una baja exigencia académica en la carrera. Pareciera ser que la exigencia por parte de los formadores y de la propia 


\section{Lobos, Muñoz, \& Valenzuela-Curiosidad Intelectual}

universidad es más baja de lo esperado. Este desencanto se produciría durante los primeros años de la carrera, en donde los estudiantes relatan que no es necesario aprender todo y que, por ende, no se precisa de curiosidad intelectual para obtener buenos resultados académicos en las carreras de pedagogía. Esto también animaría a los estudiantes a esforzarse mínimamente en las clases y evaluaciones.

Este panorama poco desafiante para los estudiantes haría que desarrollaran estrategias para obtener buenas calificaciones o "pasar los ramos" sin la necesidad de invertir un mayor esfuerzo intelectual:

Siento que los profesores no demandan tanto de los estudiantes dentro de la universidad, no le piden tanto, sino que piden el mínimo esfuerzo y los alumnos, en cierta medida, también se acostumbran o yo [...] me acostumbré a eso, casi al mínimo esfuerzo dentro de la universidad (E1).

Así, los entrevistados reconocen que esta falta de curiosidad o de interés propio se ve acrecentada a partir de un programa que ellos caracterizan como poco desafiante intelectualmente. Existe una sensación compartida de conformismo o de estancamiento intelectual que -muchas veces- acomoda a los estudiantes, pero que también los lleva a preguntarse "para qué voy a seguir con más si al final no van a preguntar más" (E6).

En este mismo sentido, se destaca en el discurso de los entrevistados la idealización del formador universitario como un profesional intelectual, culto, reflexivo o crítico, y que es parte de un ambiente reflexivo dentro de la universidad. Sin embargo, esto es algo que también se echa en falta. Parecieran no existir espacios de relación entre los actores de la comunidad en donde se conversen, reflexionen y analicen ciertos temas que pueden ser de interés para el quehacer pedagógico: "acá no ocurre nada de eso, entonces, sí, nos quedamos haciendo una vida muy cotidiana y poco académica,... poco intelectual" (E2). Esto no se refiere sólo al contexto del programa, sino que los estudiantes lo resienten también como una carencia a nivel de la institución universitaria.

En síntesis, se atribuye a un contexto académico poco desafiante o exigente o un ambiente de poca reflexión y discusión intelectual o del acontecer nacional, la causa de esta sensación de falta de desafío que señalan 
los estudiantes, y fomentaría una desvalorización de la curiosidad intelectual en los entrevistados. Así, según los propios entrevistados, este ambiente estimularía una suerte de conformismo en los estudiantes de pedagogía, y la falta de desafío, propiciaría el desarrollo de estrategias para responder a las evaluaciones, sin un mayor esfuerzo.

\section{La Docencia como un Quehacer Técnico}

Otro de los factores de desmotivación detectado en el discurso de los estudiantes entrevistados es la percepción progresiva de la pedagogía como un quehacer de carácter técnico. En este contexto, la formación docente consistiría en aprender una manera de enseñar que no implica mayores conocimientos teóricos o intelectuales. En esta perspectiva lo relevante es la práctica, el trabajo en aula y la relación con los alumnos.

Como que no importara nada más, simplemente, pareciera ser que lo que importa es aprender el cómo hacerlo, tal vez, o tener herramientas de cómo aplicar lo que uno quiere hacer dentro de la sala de clases sin detenerse demasiado en el qué, porque muchas veces el qué está entregado por los Planes y Programas, entonces, ya tienes más o menos los contenidos que debes saber, que ya es una base y de ahí en adelante importa simplemente cómo hacerlo. Y en ese sentido, creo, que se pierde esta curiosidad intelectual, porque se cree que se sabe ya lo básico para llevarlo a cabo (E1).

Según los relatos de los estudiantes, el interés pareciera focalizarse principalmente en el cómo enseñar. Dicho de otro modo, los conocimientos serían solo relevantes en la medida en que sea posible aplicarlos en sus prácticas pedagógicas. En este sentido, la dimensión académica de la formación es desvalorada o tildada de "teoría" en tono peyorativo, al punto de afirmar que, en la práctica, "nunca lo he relacionado a algún texto o alguna bibliografía" (E6).

\section{Curiosidad Intelectual ¿Para Qué?}

El relato de estos estudiantes, identificados con una baja en la curiosidad intelectual durante su formación docente, evidencia que ser curioso intelectualmente no es gravitante en su formación profesional, y que esta idea 


\section{Lobos, Muñoz, \& Valenzuela-Curiosidad Intelectual}

se instalaría ya desde primer año de formación docente. Esta falta de curiosidad tendría que ver con un desencanto con la dimensión académica intelectual de la carrera y la falta de exigencia y desafíos por parte de profesores y sus respectivas clases. Así, la ausencia de un ambiente académico que incentive la curiosidad intelectual es visto como un contexto en el cual no existiría ni la necesidad ni el interés de ir más allá o ser curioso intelectualmente, sino que habría una suerte de conformismo con lo enseñado en la universidad. En este contexto, la curiosidad o la motivación por la búsqueda de nuevos conocimientos, no tendría mayor sentido.

No se conversa de política, no se conversa de educación, no se conversa de economía, no se conversa ni siquiera del acontecer nacional o de "oye, descubrí este autor nuevo, mira, es re interesante". Tampoco se da ese intercambio entre los compañeros y tampoco se da entre los profesores, porque básicamente se lee lo mismo, nos damos vuelta en lo mismo, el mismo autor nos sirve tres, cuatro veces, por ejemplo, durante la carrera. Entonces, pareciera ser que ahí nos estancamos (E10).

\section{Discusión}

El presente estudio se focalizó en determinar si la curiosidad intelectual, como variable relevante dentro de la formación de futuros profesores, cambiaba durante el proceso de formación universitario.

Si bien en este estudio se ha observado un incremento de la curiosidad intelectual en algunos estudiantes de la muestra, al mismo tiempo se observa una baja significativa en esta variable en un número importante de estudiantes de Magisterio. La constatación de una caída negativa en esta variable nos llevó a preguntarnos por las razones de este descenso en la curiosidad intelectual en profesionales clave para el desarrollo de nuestro país, y comprender por qué se ve mermada su curiosidad intelectual en un espacio que debiera, por el contrario, desarrollarla.

La evidencia recogida en estudiantes de Magisterio que disminuyeron significativamente su curiosidad intelectual durante el proceso de formación pedagógica muestra que las causas atribuidas a esta baja estarían asociadas a) a las expectativas académicas insatisfechas de los estudiantes, b) a un 
contexto académico poco desafiante y c) a una forma más bien técnica de concebir la labor docente por una parte importante de sus formadores.

En términos concretos, los estudiantes atribuyen su desmotivación a la falta de desafío por parte de los formadores y al ambiente dentro de la carrera, la cual no contempla reflexiones o análisis intelectualmente desafiantes. A ello se suma la falta de exigencia académica de la propia carrera y la percepción de la docencia como un quehacer instrumental, en donde lo práctico se impone por sobre los conocimientos teóricos. Esto es posible de constatar en todos los entrevistados, independientemente de su antigüedad en el programa de estudios.

Estos resultados son consistentes con investigaciones que muestran que la percepción de una baja calidad de la formación y específicamente en aspectos relacionados con la exigencia, inciden en la desmotivación de los estudiantes. Ello es especialmente relevante en instituciones con baja selectividad (Ruffinelli, 2013). Si bien Chile ha impulsado políticas tendientes a aumentar los niveles de exigencia académica en la formación de profesores, esto permanece aún como un desafío.

Junto con lo anterior, las atribuciones que realizan los entrevistados respecto a esta baja en la curiosidad intelectual coinciden con dinámicas formativas que se caracterizan por insistir fuertemente en los aspectos técnicos de la docencia (Cuadra \& Catalán, 2016), descuidando aspectos más académicos de la profesión. De esta manera, nos enfrentamos a una paradoja: queriendo profesionalizar el oficio docente, logramos precisamente lo contrario (Cf. Assaél et al., 2012; Ruffinelli, 2016). Así, el paradigma actual de la Educación Superior concibe al sistema universitario como un mercado competitivo y al estudiante como un cliente, donde el valor del título profesional queda reducido a una mera inversión (Troschitz, 2017; Williams, Gómez, \& Kurkiewicz, 2013). Según Paricio (2017) en el sistema educativo la titulación se entiende como un entrenamiento para obtener éxito laboral, por lo tanto, existe un predominio de las capacidades técnicas e instrumentales en la formación, dejando de lado el pensamiento científico, la reflexión intelectual y crítica. A ello se suma el otorgamiento de un grado de licenciado, que por norma ministerial es concurrente a la obtención del título profesional (Aequalis, 2011), muchas veces con un trabajo de titulación anclado en la práctica profesional sin el desarrollo de una tesis de grado, 


\section{Lobos, Muñoz, \& Valenzuela-Curiosidad Intelectual}

minimizando el desarrollo de habilidades del pensamiento.

Lo anterior se condice con los resultados expuestos. La forma de entender la pedagogía como un quehacer técnico que exige la resolución rápida de problemas concretos y prácticos, va en desmedro del interés por la búsqueda de nuevas interrogantes, así como de nuevos conocimientos. La concepción de la pedagogía como una forma "correcta" de enseñar, deja relegado a un segundo plano los conocimientos teóricos propios de la disciplina.

Un fenómeno similar ocurre en el contexto universitario estudiado, donde ha emergido una nueva forma de comprender la relación entre estudiantes y la institución. La concepción de los estudiantes como clientes en algunas instituciones de educación superior distorsiona la relación entre profesor y estudiante, en donde el primero cumple tradicionalmente un rol de mentor. Junto con ello, también se difuminan las nociones tradicionales respecto al rol del estudiante, que dicen relación con responsabilidades, la exigencia de excelencia y el trabajo duro (Troschitz, 2017; Trout, 1997), es decir, el estudiante-cliente recibiría un producto debido a su inversión en una carrera determinada. Ello no demanda mayores esfuerzos o desafíos para cumplir las exigencias que la formación conlleva.

Este ambiente poco desafiante -también mencionado por los docentes en formación en este estudio- potencia un rol del estudiante como ente pasivo frente a los conocimientos que recibe y que propicia una disminución en su curiosidad intelectual.

Estos hallazgos constituyen una primera pista para hacernos conscientes de que muchos de nuestros estudiantes de pedagogía pierden su curiosidad intelectual durante la formación y junto con lo anterior, permiten identificar eventuales causas de este fenómeno y debiesen constituir una alerta para las instituciones con el fin de evitar que ello siga sucediendo.

Un acierto de este estudio dice relación con la coherencia de los resultados, que fueron emergiendo a partir de la convergencia entre los discursos de los futuros docentes. Así, pese a la heterogeneidad de la muestra (en cuanto a diversidad geográfica, tipo de institución y años cursados en el programa) sus explicaciones sobre las causas que influyen en la curiosidad intelectual son coincidentes.

Si bien esta investigación devela la existencia de ciertos factores que estarían afectando la curiosidad intelectual de los futuros docentes, una 
limitación está dada por el tamaño de la muestra de entrevistados. Ello hace difícil establecer generalizaciones a partir de estos resultados, no obstante, nos muestra -de forma exploratoria- situaciones que, en voz de sus actores, incidirían en un proceso de desmotivación en torno a una actitud curiosa intelectualmente en la universidad. Lo anterior nos plantea como desafío un estudio de mayor envergadura que considere también aquellos casos de alza en la curiosidad intelectual y, por otra parte, ampliar la muestra en pos de confirmar estos hallazgos.

\section{Agradecimientos}

Esta investigación ha sido financiada por la Comisión Nacional de Investigación en Ciencia y Tecnología (Conicyt-Chile) a través de los Proyectos Fondecyt Regular 1150533, 1170779 y 1181159 .

\section{References}

Aequalis (2011). Estructura de títulos y grados y el marco regulatorio asociado. In M. Jiménez, F. Lagos, \& F. Durán (Eds.), Propuestas para la Educación Superior. Foro Aequalis y las transformaciones necesarias (pp. 181-197). Santiago: Aequalis - Inacap.

Ardila, E., \& Rueda, J. (2013). La saturación teórica en la teoría fundamentada: su de-limitación en el análisis de trayectorias de vida de víctimas de desplazamiento forzado en Colombia. Revista colombiana de sociología, 32(2), 93-114. doi: 10.15446/rcs

Assaél, J., Contreras, P., Corbalán, F., Palma, E., Campos, J., Sisto, V., \& Redondo, J. (2012). Ley SEP en escuelas municipales emergentes: ¿cambios en la identidad docente? Revista de pedagogía crítica Paulo Freire, (11), 219-228. doi: 10.25074/07195532.11.453

Berlyne, D. E. (1954). A theory of human curiosity. British Journal of Psychology, 45, 180-191. doi: 10.1111/j.2044-8295.1954.tb01243.x Bouffard, T., Mariné, C., \& Chouinard, R. (2004). Interdépendance des caractéristiques individuelles et contextuelles dans la motivation à apprendre. Revue des sciences de l'éducation, 30(1), 3-8. Retrieved from https:/www.erudit.org/fr/revues/rse/2004-v30-n1rse962/011766ar/ 
42 Lobos, Muñoz, \& Valenzuela-Curiosidad Intelectual

Costa, A. L., \& Kallick, B. (2009). Habits of mind across the curriculum: Practical and creative strategies for teachers. Alexandria, VA: ASCD.

Cuadra, D., \& Catalán, J. (2016). Teorías subjetivas en profesores y su formación profesional. Revista Brasileira de Educação, 21(65), 299324. doi: 10.1590/S1413-24782016216517

Day, C., \& Gu, Q. (2012). Profesores: vidas nuevas, verdades antiguas: una influencia decisiva en la vida de los alumnos (vol. 121). Madrid: Narcea Ediciones.

Delors, J. (2013). The treasure within: Learning to know, learning to do, learning to live together and learning to be. What is the value of that treasure 15 years after its publication? International Review of Education, 59, 319-330. doi: 10.1007/s11159-013-9350-8

Delors, J., Al Mufti, I., Amagi, I., Carneiro, R., Chung, F., Geremek, B., \& Nazhao, Z. (1996). Informe a la Unesco de la comisión internacional sobre la educación para el siglo XXI: La educación encierra un tesoro. Madrid: Santillana, Ediciones UNESCO.

Demirdag, S. (2015). The relationship between critical thinking abilities and classroom management skills of high school teachers. Educational Research and Reviews, 10(7), 850-855. doi: 10.5897/ERR2015.2173

Falardeau, É., \& Simard, D. (2007a). Le rapport à la culture des enseignants de français et son rôle dans l'articulation de la culture avec les contenus disciplinaires. In E. Falardeau, C. Fisher, D. Simard, \& N. Sorin (Eds.), La didactique du français. Les voies actuelles de la recherche (pp. 147-163). Villeneuve d'Ascq: Presses universitaires du Septentrion.

Falardeau, É., \& Simard, D. (2007b). Rapport à la culture et approche culturelle de l'enseignement. Canadian Journal of Education/Revue canadienne de l'éducation, 30(1), 1-24. doi: 10.2307/20466623

Flanagan, A., \& Arancibia, V. (2005). Talento académico: Un análisis de la identificación de alumnos talentosos efectuada por profesores. Psykhe, 1(14), 121-135. doi: 10.4067/S0718-22282005000100010 
González, J. H. (2006). Discernimiento: Evolución del Pensamiento Crítico en la educación superior, el proyecto educativo de la Universidad Icesi. Cali: Universidad Icesi.

Lauriola, M., Litman, J. A., Mussel, P., De Santis, R., Crowson, H. M., \& Hoffman, R. R. (2015). Epistemic curiosity and self-regulation. Personality and Individual Differences, 83, 202-207. doi: 10.1016/j.paid.2015.04.017

MINEDUC. (2019). Beca Vocación de profesor. Retrieved from https://www.ayudamineduc.cl/ficha/beca-vocacion-de-profesorpedagogias-5

Noordewier, M. K., \& van Dijk, E. (2017). Curiosity and time: from not knowing to almost knowing. Cognition and Emotion, 31(3), 411-421. doi: 10.1080/02699931.2015.1122577

Oliva, M. A. (2017). Architecture of Chilean educational policy (19902014): The Curriculum, a place of metaphors. Revista Brasileira de Educação, 22(69), 405-428. doi: 10.1590/S1413-24782017226921

Paricio, J. (2017). El estudiante como cliente: un cambio de paradigma en la educación superior. Debats. Revista de cultura, poder y sociedad, 131(2), 41-55. doi: 10.28939/iam.debats.131-2.4

Powell, C., Nettelbeck, T., \& Burns, N. R. (2016). Deconstructing intellectual curiosity. Personality and Individual Differences, 95, 147-151. doi: 10.1016/j.paid.2016.02.037

Powell, C., Nettelbeck, T., \& Burns, N. R. (2017). The incremental validity of intellectual curiosity and confidence for predicting academic performance in advanced tertiary students. Personality and Individual Differences, 116, 51-56. doi: 10.1016/j.paid.2017.04.011

Precht, A., Valenzuela, J., Muñoz, C., \& Sepúlveda, K. (2016). Familia y motivación escolar: desafíos para la formación inicial docente. Estudios pedagógicos (Valdivia), 42(4), 165-182. doi: 10.4067/S0718-07052016000500010

Rosas, R., \& Santa Cruz, C. (2013). Dime en qué colegio estudiaste y te diré qué CI tienes: Radiografía al desigual acceso al capital cognitivo en Chile. Santiago: Ediciones UC. 
44 Lobos, Muñoz, \& Valenzuela-Curiosidad Intelectual

Ruffinelli, A. (2013). La calidad de la formación inicial docente en Chile: la perspectiva de los profesores principiantes. Calidad en la educación, (39), 117-154. doi: 10.4067/S0718-45652013000200005

Ruffinelli, A. (2016). Ley de desarrollo profesional docente en Chile: de la precarización sistemática a los logros, avances y desafíos pendientes para la profesionalización. Estudios pedagógicos (Valdivia), 42(4), 261-279. doi: 10.4067/S0718-07052016000500015

Ryan, R. M., \& Deci, E. L. (2017). Self-Determination Theory. Basic Psychological Needs in Motivation, Development, and Wellness. New York: Guilford Press.

Sanz, R., Bertolín, A. G., \& Lujan, E. L. (2017). La excelencia docente: una mirada desde la pedagogía. Edetania: estudios y propuestas socioeducativas, (52), 219-241. Retrieved from http://revistas.ucv.es/index.php/Edetania/article/view/329

Servicio de Información de Educación Superior. (2016). Retención de ler año de pregrado Cohortes 2011-2015. Retrieved from http://tinyurl.com/retencion2016-informe

Siegle, D., Rubenstein, L. D., \& Mitchell, M. S. (2014). Honors students' perceptions of their high school experiences: The influence of teachers on student motivation. Gifted child quarterly, 58(1), 35-50. doi: 10.1177/0016986213513496

Silverman, L. K. (1997). Family counseling with the gifted. In N. Colangelo \& G. Davis (Eds.), Handbook of gifted education (2 ed., pp. 382-397). Boston: Allyn \& Bacon.

Silvia, P. J. (2012). Curiosity and motivation. In R. M. Ryan (Ed.), Oxford handbook of motivation (pp. 157-166). New York: Oxford University Press.

Silvia, P. J., \& Kashdan, T. B. (2009). Interesting things and curious people: Exploration and engagement as transient states and enduring strengths. Social and Personality Psychology Compass, 3(5), 785797. doi: 10.1111/j.1751-9004.2009.00210.x

Todorescu, L.-L., Popescu-Mitroi, M.-M., \& Greculescu, A. (2015).

Students' Views on the Teacher as An Information Provider. Procedia - Social and Behavioral Sciences, 197, 184-189. doi: 10.1016/j.sbspro.2015.07.121 
Troschitz, R. (2017). Higher Education and the Student: From welfare state to neoliberalism. London: Routledge.

Trout, P. A. (1997). Disengaged students and the decline of academic standards. Academic Questions, 10(2), 46-56. doi: 10.1007/s12129997-1067-3

Valenzuela, J., Muñoz, C., Precht, A., Silva Peña, I., Marfull-Jensen, M., \& Oliva Martínez, J. (2018). Trayectorias motivacionales en la formación pedagógica: motivación y desmotivación en la Formación Inicial Docente (FID) de futuros profesores de Educación Básica (Informe Final Fondecyt Regular 1150533). Santiago: Fondo Nacional de Desarrollo Científico y Tecnológico.

Valenzuela, J., Muñoz, C., Precht, A., Silva-Peña, I., Oliva, M. A., \& Marfull-Jensen, M. (2016). Inventario Motivacional para la Formación Docente (IMFD). Viña del Mar: Centro de Estudios Avanzados, Universidad de Playa Ancha.

Viau, R. (2009). La motivation en contexte scolaire. Bruxelles: De Boeck.

Viau, R. (2013). La motivation à apprendre des étudiants mieux comprendre pour mieux agir. [Conference à l'Université Catholique de Lyon]. Retrieved from https://www.youtube.com/watch?v=30h3qjai9I

Von Stumm, S., Hell, B., \& Chamorro-Premuzic, T. (2011). The hungry mind: Intellectual curiosity is the third pillar of academic performance. Perspectives on Psychological Science, 6(6), 574-588. doi: 10.1177/1745691611421204

Watson, L. (2017). Why should we educate for inquisitiveness? In J. Baehr (Ed.), Intellectual Virtues and Education (pp. 38-53). New York: Routledge.

Williams, M. N., Gómez, C. A., \& Kurkiewicz, D. (2013). Assumptions of multiple regression: correcting two misconceptions. Practical assessment, research \& evaluation, 18(11), 1-14. Retrieved from https://scholarworks.umass.edu/pare/vol18/iss1/11/

Zakhartchouk, J.-M. (1999). L'enseignant, un passeur culturel. Paris: ESF. 
46 Lobos, Muñoz, \& Valenzuela-Curiosidad Intelectual

\section{Constanza Lobos Universidad Alberto Hurtado}

Carla Muñoz Departamento de Psicología de la Universidad Católica del Maule

Jorge Valenzuela Facultad de Ciencias de la Educación de la Universidad Católica del Maule

Contact Address: Universidad Alberto Hutado. Av Libertador Bernardo O'Higgins 1869, Santiago, Región Metropolitana, Chile.

Email: coni.lobosguerrero@gmail.com 